Mappemonde

Revue trimestrielle sur l'image géographique et les formes du territoire

$119 \mid 2017$

Varia

\title{
Commentaire de la carte géomorphologique du bassin versant du Brunet
}

Nicolas Bollot

(2) OpenEdition

Journals

Édition électronique

URL : http://journals.openedition.org/mappemonde/2387

DOI : $10.4000 /$ mappemonde.2387

ISSN : 1769-7298

Éditeur

UMR ESPACE

Référence électronique

Nicolas Bollot, «Commentaire de la carte géomorphologique du bassin versant du Brunet »,

Mappemonde [En ligne], 119 | 2017, mis en ligne le 01 janvier 2017, consulté le 15 septembre 2020.

URL : http://journals.openedition.org/mappemonde/2387

Ce document a été généré automatiquement le 15 septembre 2020

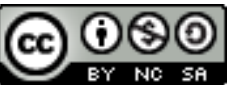

La revue Mappemonde est mise à disposition selon les termes de la Licence Creative Commons Attribution - Pas d'Utilisation Commerciale - Partage dans les Mêmes Conditions 4.0 International. 


\title{
Commentaire de la carte géomorphologique du bassin versant du Brunet
}

\author{
Nicolas Bollot
}

Références de la thèse

воLLOт N. (2014). Les mouvements de terrain du vignoble champenois : approche géomorphologique et hydrologique. Thèse de doctorat en Géographie physique, humaine, économique et régionale, Université de Reims Champagne-Ardenne, 2 tomes, $343 \mathrm{p}$.

\section{Introduction}

1 Malgré des reliefs de faible énergie, les versants du vignoble champenois sont affectés par de très nombreux glissements de terrain. Dans un secteur où les enjeux économiques sont forts, ces glissements provoquent de coûteux dégâts. Dans ce cadre, la cartographie géomorphologique de l'ensemble du secteur d'étude s'avère nécessaire pour identifier, spatialiser et donc comprendre le processus. 
Carte géomorphologique du bassin versant du Brunet

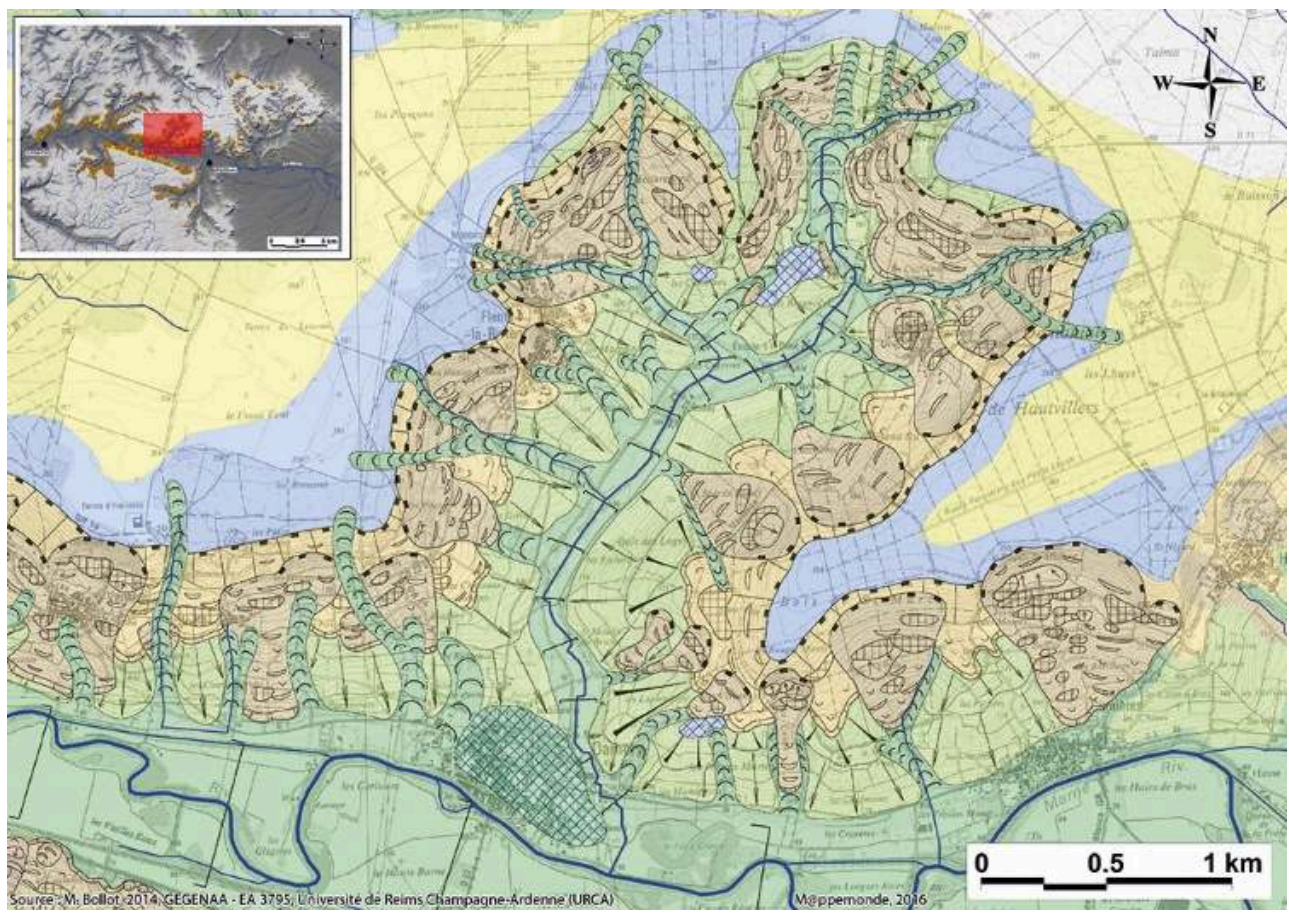

Source: M. Bollot, 2014, GEGENAA - EA 3795, Université de Reims Champagne-Ardenne (URCA)

\section{Méthodologie}

Les cartes sont dressées selon la méthode champenoise (Guérémy et Marre, 1996) partiellement modifiée. Basée sur la dynamique des différents processus, cette méthode est bien adaptée à l'étude des aléas naturels, dont les glissements de terrain. Une gamme de couleurs est ainsi appliquée au processus glissement de terrain pour permettre une lecture rapide. Les valeurs de couleurs diffèrent ensuite selon le degré d'activité du processus: claire pour les processus stables, plus foncée pour les phénomènes dormants et foncée pour ceux qui sont actifs (figure 1). 
Figure 1. Structure de la méthode champenoise de cartographie géomorphologique

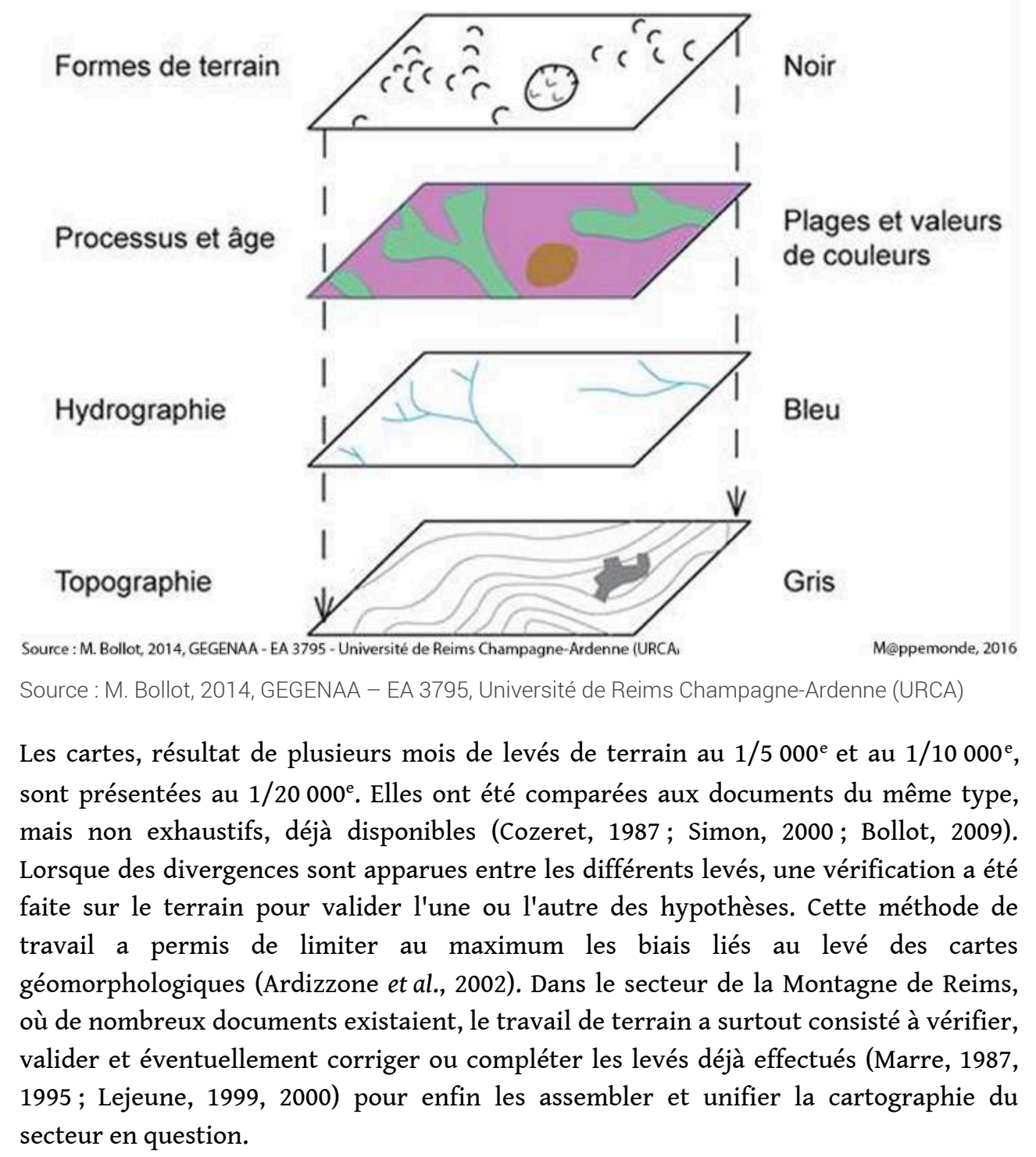

\section{Étude de cas : le bassin versant du Brunet}

4 Le Brunet, en rive droite, est le premier affuent majeur de la Marne en aval de l'entonnoir de percée cataclinale. Il la rejoint par une vallée à fond plat de direction globalement nord-sud.

Dans l'ensemble de la vallée du Brunet, la craie affeure jusqu'à la cote 150 mètres environ. Elle engendre des bas de versant en pente forte se raccordant à un fond de vallée plat (figure 2). Dans la partie amont du bassin, la partie supérieure des versants de rive droite présente un profil réglé visible au nord de Fleury-la-Rivière et à Cormoyeux. Il se prolonge par un niveau de glacis qui se raccorde à un replat situé à une altitude d'environ 175 mètres correspondant au toit des argiles de l'Yprésien inférieur. Il ne reste aujourd'hui que deux lambeaux observables de ces glacis, aux «Fourtières » et vers le village de Cormoyeux. Le premier rejoint un replat au lieu-dit 
«Les Chêneaux", raccordable à celui des «Brugnodes» à l'est. Le second est aujourd'hui tronqué par l'incision du ruisseau des Gros Sourdons.

Figure 2. Fond de la vallée du Brunet à l'aval du bassin versant

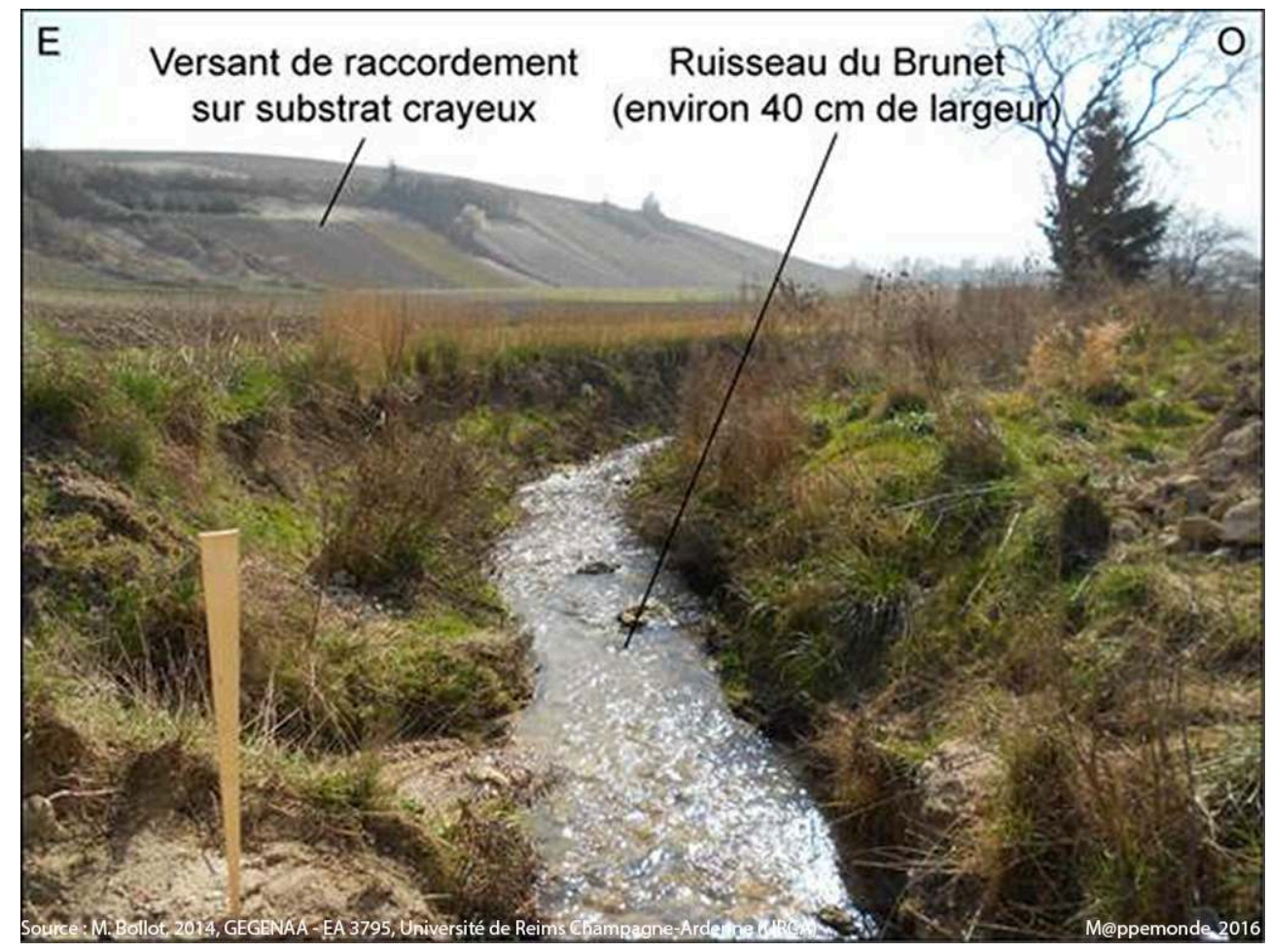

Source: M. Bollot, 2014, GEGENAA - EA 3795, Université de Reims Champagne-Ardenne (URCA)

6 Dans la section aval du bassin du Brunet et jusqu'à la confuence avec la Marne, la partie supérieure des versants est affectée par des instabilités gravitaires dont le modelé caractéristique se propage jusqu'au niveau du replat, situé à environ 175 mètres d'altitude, sous la forme de fronts de coulée. Bien que certains replats soient encore visibles en aval du bassin versant, aux « Bois des Loges » ou aux "Chéneaux Rouges », les modelés de glissements sont estompés : les escarpements de tête sont régularisés et affectés localement par de la solifuxion, et les coulées sont recouvertes par des dépôts colluviaux.

7 Dans la continuité spatiale des très vieux glissements de terrain, un modelé de glissement plus récent et peu régularisé s'identifie de part et d'autre de la vallée. En amont du bassin, sur les versants de Cormoyeux et du nord de Romery, ces glissements occupent l'ensemble de la partie médiane du versant. L'escarpement de tête est continu entre les différents vallons provenant du plateau. Les replats ont une forme longiligne et n'occupent le versant qu'avec parcimonie. Les plus importants se situent aux "Caillaudries » où un glissement se prolonge par une coulée dont le bourrelet frontal est plus marqué et s'est propagé plus bas sur le versant. Ce type de glissement est également présent au nord de la commune de Fleury-la-Rivière sur le versant ouest, aux lieux-dits "Les Beauregards » ou "Les Chenillonnes ", où le modelé de glissement est encore plus marqué et les coulées plus longues (figure 3). Des réactivations actuelles ou récentes affectent ces glissements, notamment au niveau de l'escarpement 
de tête des «Vieilleries». Sur les bas versants des "Brugnodes", deux petits glissements peu profonds se sont déclenchés récemment.

Figure 3. Escarpement de tête sur le versant de rive droite du Brunet à Fleury-la-Rivière

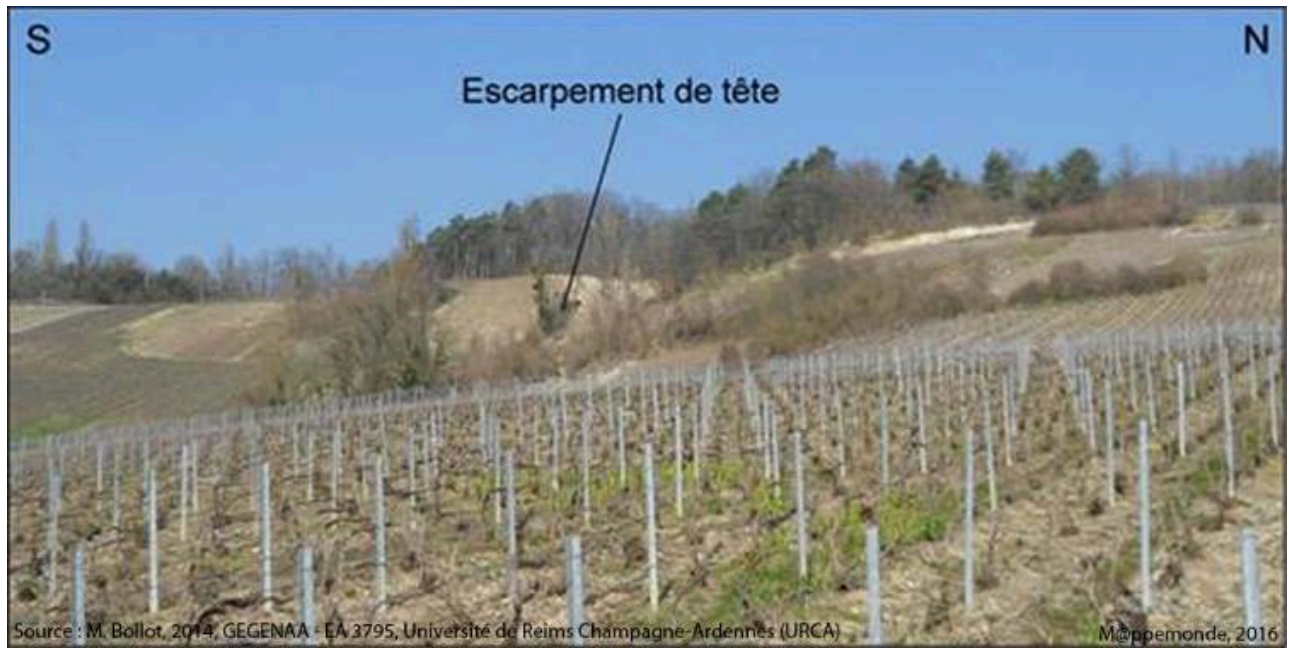

Source : M. Bollot, 2014, GEGENAA - EA 3795, Université de Reims Champagne-Ardenne (URCA)

8 La même relation spatiale entre glissements anciens et récents s'observe dans la partie aval du bassin versant de Brunet, mais la morphologie diffère un peu. L'escarpement de tête du glissement de Romery, vers 240 mètres d'altitude, est circulaire. Le glissement s'étend ensuite jusqu'au village, installé sur un replat au sein de la coulée. À ce niveau, le glissement s'évase jusqu'au fond de la vallée après un segment plus étroit. Si l'ampleur du glissement de Romery est beaucoup plus grande - entre deux et trois fois plus d'emprise surfacique -, sa morphologie rappelle, au même titre que le glissement du Moulin de Brunet plus en aval, celle des glissements de la vallée du Cubray. Les autres glissements de terrain de la partie aval du bassin versant de Brunet, et notamment ceux du versant oriental, naissent également vers 240 mètres d'altitude. Ils présentent un escarpement de tête circulaire atteignant 50 mètres de commandement. Celui $\mathrm{du}$ «Bois de Raday» se prolonge par une contrepente aux rebords abrupts qui prouve le caractère rotationnel du déplacement. Les coulées s'engouffrent ensuite dans des vallons et s'amincissent, sans toutefois atteindre la plaine alluviale. Ces glissements sont donc profonds, rotationnels et systématiquement placés en tête de vallon.

Proche de l'exutoire, la rive droite du Brunet, où est implanté le village de Damery, se présente comme une zone plane perchée de 10 à 15 mètres au-dessus du talweg. Cette forme est associée à un lambeau de cône de déjection du Brunet sur la plaine alluviale de la Marne (Simon, 1999). Le cours d'eau actuel, très peu puissant (figure 2), est ainsi détourné vers l'est via un fond de vallée moins large.

\section{Conclusion}

Le levé cartographique permet donc d'inventorier et caractériser les glissements de terrain, processus dominant d'évolution des versants du vignoble champenois. Plusieurs niveaux d'activités sont décelés. Des formes stabilisées et en partie régularisées sont fréquentes dans la partie haute des versants. Elles peuvent être recoupées par des glissements plus récents, parfois plus volumineux, qui peuvent se 
propager jusqu'au fond des vallées. Leurs formes, encore bien identifiables sur les versants, semblent néanmoins figées, ce qui permet de considérer ces glissements comme « dormants ». Enfin, des phénomènes d'ampleur moindre, souvent superficiels, traduisent une activité récente ou actuelle. Ces petits glissements sont généralement des réactivations partielles des plus anciens. L'ensemble de ces résultats complète et confirme ceux obtenus ou soupçonnés dans les études précédentes. Base d'une typologie et d'un travail d'analyse spatiale, la cartographie géomorphologique apparait ainsi comme un une étape essentielle pour comprendre l'apparition et l'évolution des glissements de terrain du vignoble champenois.

\section{BIBLIOGRAPHIE}

ARDIZZONE F., CARDINALI M., CARRARA A., GUZZETTI F., REICHENBACH P. (2002). « Impact of mapping errors on the reliability of landslide hazard maps ». Natural Hazards and Earth System Sciences, $\mathrm{n}^{\circ} 2$, p. 3-14.

BOLLOT N. (2009). Cartographie et étude des glissements de terrain sur le territoire des communes de PassyGrigny, Vandières, Verneuil et Vincelles. Mémoire de Master 1, Université de Reims ChampagneArdenne, $59 \mathrm{p}$.

COZERET O. (1987). « Les mouvements de terrain dans le secteur de Champillon - Dizy - Hautvillers (Marne)». Travaux de l'Institut de Géographie de Reims, n 69-72, p. 129-150.

GUÉRÉMY P., MARRE A. (1996). « Une nouvelle méthode de cartographie géomorphologique applicable aux aléas naturels ». Travaux de l'Institut de Géographie de Reims, n 93-94, p. 5-40.

LEJEUNE O. (1999). Les mouvements de terrain sur le flanc septentrional de la Montagne de Reims entre la commune de Verzenay et le lieu-dit le Mont Tournant. Mémoire de maîtrise Université de Reims, $140 \mathrm{p}$.

LEJEUNE O. (2000). Le karst et les mouvements de terrain sur le versant nord de la Montagne de Reims de Villers-Allerand au Mont Tournant. Mémoire de DEA, Université de Lille 1, 129 p.

MARRE A. (1987). « Le mouvement de terrain de Rilly-la-Montagne du 23 août 1986, naissance et évolution ». Travaux de l'Institut de Géographie de Reims, nº 69-72, p. 95-111.

MARRE A. (1995). Les mouvements de terrain sur les communes de Bouzy et d'Ambonnay. Rapport d'activité, Université de Reims Champagne-Ardenne, GRECA Reims, 18 p.

SIMON F. (1999). La vallée du Ru du Brunet (Marne) : la disproportion entre la taille de la vallée et celle du Ruisseau (étude de la morphogénèse). Mémoire de maîtrise, Institut de Géographie, Université de Reims, 157 p.

SIMON F. (2000). Les glissements de terrain affectant le versant nord de la vallée de la Marne entre Ambonnay et Barzy-sur-Marne (Champagne, France). Synthèse. Mémoire de DEA, Université des Sciences et Technologies de Lille, $262 \mathrm{p}$. 


\section{ANNEXES}

Légende des cartes géomorphologiques produites dans la thèse

\begin{tabular}{|c|c|c|c|}
\hline \multicolumn{4}{|l|}{ Formes } \\
\hline M & Glacis & 6 & Escarpement de tửe \\
\hline & Versant réglé & 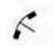 & $\begin{array}{l}\text { Escarpement de tête } \\
\text { regularise }\end{array}$ \\
\hline & $\begin{array}{l}\text { Versant de } \\
\text { raccordenent }\end{array}$ & 6 & $\begin{array}{l}\text { Escarpement de tête } \\
\text { inferieur a } 10 \mathrm{~m}\end{array}$ \\
\hline$\otimes$ & Replat & $\kappa$ & $\begin{array}{l}\text { Escarpement de tête } \\
\text { inférieur à } 5 \mathrm{~m}\end{array}$ \\
\hline , & Vallce à fond plat & $\theta$ & Replat de glissement \\
\hline ב, & Vallike en berceau & Q & Contrepente \\
\hline & Vallée en avm & $\because$ & $\begin{array}{l}\text { Solifluxion } \\
\text { Coulke }\end{array}$ \\
\hline$\underset{\otimes}{\langle 1\rangle}$ & Cône de déjection & & Bourrelet frontal \\
\hline
\end{tabular}

\section{Processus}

$\square$ Ruissellement diffus
$\square$ Ruissellement concentré
$\square$ Ruissellement concentré ancien
$\square$ Actions ćliennes
$\square$ Altération chimique
$\square$ Domaine anthropique

Autre

$\frown$ Cours d'eau

Source: M. Bollot, 2014, GEGENAA - EA 3795, Université de Reims Champagne-Ardenne (URCA)

INDEX

Mots-clés : prix de thèse 2015

\section{AUTEUR}

NICOLAS BOLLOT

Université de Reims Champagne-Ardenne 